\title{
Basic Physiological Research on the Wing Flapping of the Sweet Potato Hawkmoth Using Multimedia
}

\author{
Isao Nakajima ${ }^{1,3 *}$, Yukako Yagi $^{2}$
}

\begin{abstract}
We have developed a device for recording biological data by inserting three electrodes and a needle with an angular velocity sensor into the moth for the purpose of measuring the electromyogram of the flapping and the corresponding lift force. With this measurement, it is possible to evaluate the moth-physiological function of moths, and the amount of pesticides that insects are exposed to (currently LD50-based standards), especially the amount of chronic low-concentration exposure, can be reduced the dose. We measured and recorded 2-channel electromyography (EMG) and angular velocity corresponding to pitch angle (pitch-like angle) associated with wing flapping for 100 sweet potato hawkmoths (50 females and 50 males) with the animals suspended and constrained in air. Overall, the angular velocity and amplitude of EMG signals demonstrated high correlation, with a correlation coefficient of $\mathrm{R}=0.792$. In contrast, the results of analysis performed on the peak-to-peak (PP) EMG intervals, which correspond to the RR intervals of ECG signals, indicated a correlation between $\Delta \mathrm{F}$ fluctuation and angular velocity of $\mathrm{R}=0.379$. Thus, the accuracy of the regression curve was relatively poor. Using a DC amplification circuit without capacitive coupling as the EMG amplification circuit, we confirmed that the baseline changes at the gear change point of wing flapping. The following formula gives the lift provided by the wing: angular velocity $\times$ thoracic weight - air resistance - (eddy resistance due to turbulence). In future studies, we plan to attach a micro radio transmitter to the moths to gather data on potential energy, kinetic energy, and displacement during free flight for analysis. Such physiological functional evaluations of moths may alleviate damage to insect health due to repeated exposure to multiple agrochemicals and may lead to significant changes in the toxicity standards, which are currently based on LD50 values
\end{abstract}

Key Words: Chronic low dose exposure, Physiological functional evaluation, Electromyography, LD50-based standards.

\section{BACKGROUND}

Insects, particularly species of large moths, are in danger of disappearing from the natural world. A key factor underlying this peril may be the significant impact of repeated exposure to multiple chemicals. Our concern-an assumption at this point-is that the current practice of judging acute toxicity, as represented by the use of LD50 values, is disproportionately damaging biodiversity of large insect species. We launched this basic research with the goal of screening for subtle physiological anomalies [1], [2], [3], [4].

It is conceivable that the repeated use of multiple agrochemicals will eventually cause irreparable damage to mankind.

\subsection{Repeated exposure to multiple agrochemicals}

Large moths, which travel great distances in the natural environment, are highly likely to be repeatedly exposed to multiple types of agrochemicals. While LD50 values are standard indicators of acute exposure, chronic exposure is assumed as 1/100 of the LD50 value. Simple arithmetic indicates if an individual organism is exposed to 20 different types of agrochemicals, theoretical exposure to toxins polluting the environment is $1 / 100 \times 20=1 / 5$ of the LD50 dose. Our team has cast doubts on such a simplified method of calculating chronic toxicity levels based on LD50. Our study seeks to obtain physiological data of moth flight to screen basically healthy individual specimens for those exhibiting physiological anomalies in physiological function at low level exposures, with the goal of identifying an indicator that may replace $1 / 100$ of the LD50 value.

Manuscript received June 20, 2020; Revised June 23, 2020; Accepted June 25, 2020. (ID No. JMIS-20M-06-018)

Corresponding Author (*): Isao Nakajima, Seisa University, Satsukigaoka 8-80, Aobaku,Yokohama, Japan, +81-90-8850-

8380,jh1rnz@aol.com.

${ }^{1}$ Nakajima labo., Seisa University, Yokohama, Japan. E-mail: i_nakajima@seisa.ac.jp

${ }^{2}$ Yukako Yagi, Memorial Sloan Kettering Cancer Center, New York, NY, USA, yagiy@mskcc.org

${ }^{3}$ Nakajima labo., Dept. of EMS, Tokai University School of Medicine, Isehara, Japan, ja9eco@gmail.com 


\subsection{Analysis of flapping motion}

Many reports of studies of flapping wings have focused on the theme "why insects can fly." In recent years, military research has investigated insect flight mechanisms as potential propulsion mechanisms for micro air vehicles (MAVs). A mainstream trend in the fluid dynamics approach to the study of flight mechanisms applies numerical simulation techniques to analyze the lift provided by wings. These studies typically assume the steady-state wing theory, while taking into account the additional effects such as separation vortex motion [4]-[9].

Table 1. Repeated exposure to multiple agrochemicals.

\begin{tabular}{|c|c|}
\hline 1. Insecticides & $\begin{array}{l}\text { Chemicals used to control insect } \\
\text { pests detrimental to crops }\end{array}$ \\
\hline 2. Fungicides & $\begin{array}{l}\text { Chemicals used to prevent diseases } \\
\text { detrimental to crops }\end{array}$ \\
\hline $\begin{array}{l}\text { 3. Insecticide- } \\
\text { fungicide } \\
\text { mixtures }\end{array}$ & $\begin{array}{l}\text { Chemicals used to control insect } \\
\text { pests and diseases simultaneously }\end{array}$ \\
\hline 4. Herbicides & Chemicals used to control weeds \\
\hline 5. Rodenticides & $\begin{array}{l}\text { Chemicals used to control rodent } \\
\text { pests, such as field rats, that are } \\
\text { detrimental to crops }\end{array}$ \\
\hline $\begin{array}{l}\text { 6. Plant growth } \\
\text { regulator }\end{array}$ & $\begin{array}{l}\text { Chemicals that promote or inhibit } \\
\text { the growth of crops }\end{array}$ \\
\hline 7. Attractant & $\begin{array}{l}\text { Chemicals that lure and trap mainly } \\
\text { insect pests with smell, etc. }\end{array}$ \\
\hline $\begin{array}{l}\text { 8. Spreading } \\
\text { agent }\end{array}$ & $\begin{array}{l}\text { Chemicals mixed with other } \\
\text { agrochemicals to enhance adhesion } \\
\text { to plant surfaces }\end{array}$ \\
\hline $\begin{array}{l}\text { 9. Microbial } \\
\text { pesticides }\end{array}$ & $\begin{array}{l}\text { Chemicals used to control insect } \\
\text { pests and diseases detrimental to } \\
\text { crops using microbes }\end{array}$ \\
\hline
\end{tabular}

The following equation gives the lift L produced by the translational motion of wings required to achieve airspeed $\mathrm{U}$ :

where

$$
\mathrm{L}=1 / 2\left(\rho \mathrm{U}^{2} \mathrm{SC}\right),
$$

$\mathrm{C}$ is the coefficient of lift;

$\rho$ is air density; and

$\mathrm{S}$ is the surface area of the wing.

This equation describes only a relationship that exists at a momentary snapshot of wing motion meeting a specific criterion (steady-state wing condition). Accounts based on pioneering studies and experiments suggest that for continuous wing flapping, the lift created by the second and third flap differs from that of the first flap. For this study, we decided to attach a monoaxial (mainly pitch axis) angular velocity sensor to a moth, albeit under restrictive conditions wherein the moth was suspended and constrained by a string.
We focused on angular velocity because we thought that due to its correlation with lift, we could observe muscular contraction by measuring angular velocity. This is because the measurement of the angular velocity has been technically completed in the past by measuring the force emitted by the flight of the whooper swan and the heart of the chicken (proportional to blood pressure) [10]-[12].

\section{METHODS}

\subsection{Target insect of experiment}

We gathered and analyzed physiological data of wing flapping for 100 hawkmoth (Agrius convolvuli) specimens suspended in air using string (in a constrained state).

Number of moth specimens: 100 specimens of Agrius convolvuli (50 males and 50 females)

Mean body weight: $1.1 \mathrm{~g}$

Mean dry weight: $0.28 \mathrm{~g}$ (approximately $75 \%$ of the body weight is water)

Agrius convolvuli is the largest hawkmoth found in the Japanese islands. Its wingspan may reach $105 \mathrm{~mm}$, often resulting in its being mistaken for a bird. Larvae feed on the leaves of plants belonging to the Convolvulaceae family, such as sweet potatoes, making them despised pests among farmers. They are bred for research purposes at the University of Tokyo and other institutions [13]-[15].

We selected the hawkmoth for our study due to its maximum payload, which is regarded to be around $0.3 \mathrm{~g}$. This allows these insects to carry a micro radio transmitter while flying freely outdoors in experiments to be carried out after obtaining the appropriate radio transmission license from the Ministry of Internal Affairs and Communications.

\subsection{Anesthesia}

As with vertebrates, surgical procedures on moths require anesthesia. Having discovered early that using nerve-block agents such as xylocaine acting directly on the nerves results in total loss of the moth's ability to fly, we were compelled to examine other reversible procedures.

Reducing temperatures to $8-10{ }^{\circ} \mathrm{C}$ is known to slow moth metabolism, inducing a state of suspended animation. We placed a moth in a Tupperware ${ }^{\circledR}$ with 10 small holes for ventilation and stored the container in a commercial refrigerator. The moth became completely immobile when left in the refrigerator for approximately 60 minutes, giving us time to perform a 5-minute surgical procedure. After the procedure, the moth was gradually returned to room temperature and able to fly freely in about an hour.

\subsection{Surgical procedures}


Moths are covered by generous quantities of lamellar and piriform scales. Some procedures involve preparing moths by removing the scales using air sprays to expose the exoskeleton. Based on our extensive experience with surgical techniques, we chose to secure the field of view under a surgical microscope by wetting the surgical field with a diluted antiseptic agent in preparation for the surgical procedure.

The surgical procedure involves embedding multiple electrodes into the body of the moth. For each electrode, a small hole is formed in the dorsal surface of the hard, shelllike exoskeleton using a pin fixed to a celluloid plate. The hole measures approximately $5 \mathrm{~mm}$ deep and $0.2 \mathrm{~mm}$ in diameter. The procedure does not involve bleeding. After the hole is formed, a $0.18-\mathrm{mm}$ enameled wire prepared beforehand is bent at a 90-degree angle at the tip to allow electrode insertion into the hole to a depth of approximately $4 \mathrm{~mm}$. Solder is applied beforehand to the $2-\mathrm{mm}$ section at the tip of the enameled wire.

The enameled wire must be fixed to the hole to prevent detachment during wing flapping. We applied small amounts of Aron Alpha ${ }^{\circledR}$ instant adhesive (Toagosei Co., Ltd.) for this purpose. (The team led by Prof. Ando used beeswax.) The instant adhesive has the advantage of solidifying together with the piriform scales surrounding the hole, which serve as auxiliary structures for fixation (Figure 1).

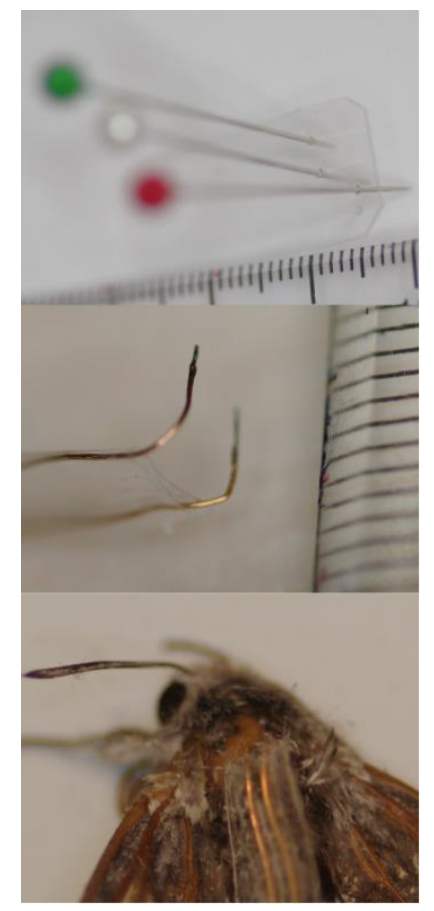

Fig. 1. Inserting and fixing electrodes for EMG.

\subsection{Basic anatomy}

The muscles involved in wing flapping are the dorsal longitudinal muscle (DLM) for the downstroke and the dorsal ventral muscle (DVM) for the upstroke. These two muscles are called power muscles, but indirect muscles. This is because the curved plate on the thoracic back is deformed and the wings are moved based on the lever principle.

The 3rd axillary muscle is responsible for wing retraction and the subtrochanteric muscle of the wing for abduction and downstroke, these two are called steering muscles, direct muscles those are linking with wings. In this study, we intended to measure two opposing muscles associated with the highest potentials (DLM and DVM) in Figure 2.

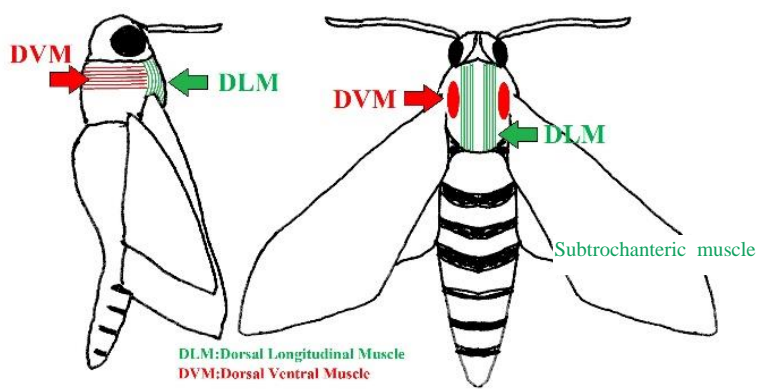

Fig. 2. Anatomical conceptual diagram of muscles involved in wing flapping.

\subsection{Hardware}

We recorded EMG and angular velocity (force) of wing flapping together with high-speed video of the moth suspended in air and constrained by string. Figure 3 presents a conceptual diagram of the experiment. The angular velocity sensor is grounded via the pin, which also serves as the fourth electrode (neutral point) of the EMG amplification circuit. The wiring to the angular velocity sensor consists of three $0.18-\mathrm{mm}$ enameled wires, connected perpendicular to the pitch angle so as not to interfere with pitch angle measurements.
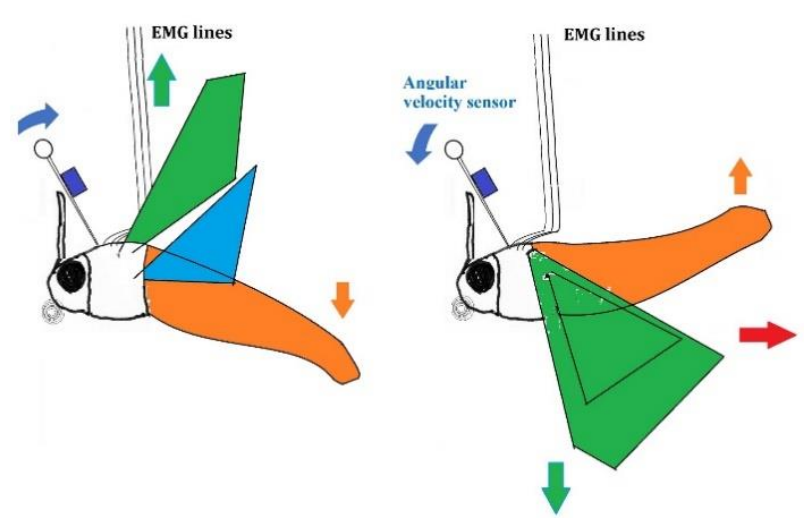

Fig. 3. Conceptual diagram of experiment. 
The moth is suspended in air and constrained with an enameled wire. However, it can still flap its wings and the counteraction of the flapping motion will manifest as vertical vibrations of the moth's abdomen to retain balance. This movement can be measured on the thorax and can be recorded as pitch angle using the angular velocity sensor.

\subsection{Circuit diagram}

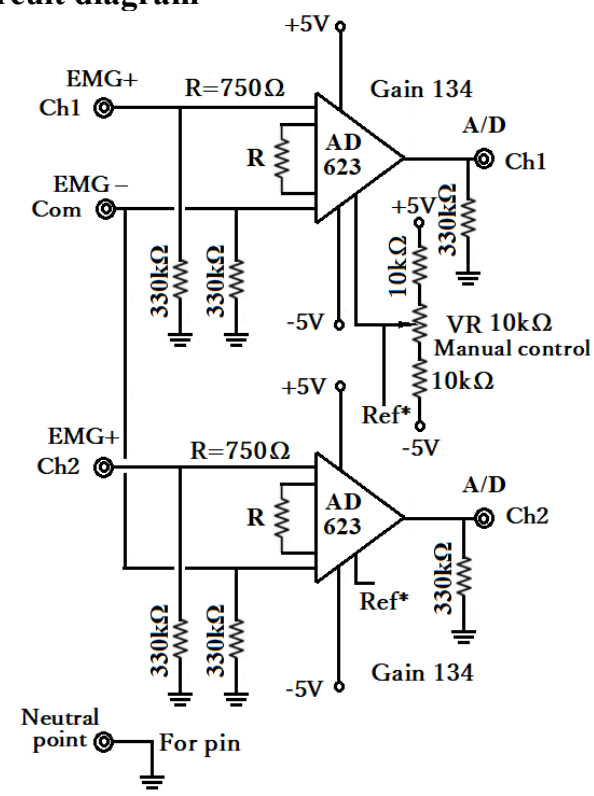

Fig. 4. Circuit diagram of EMG with two instrumentation operational amplifiers (OP AMP AD623) [16].

Since the neutral point is grounded, the zero potential of the operational amplifier is adjusted manually after inserting the electrode. Once this is adjusted, the output should remain within the range of -5 to +5 when the amplification factor (gain) is 134 and fall within the range of the A/D conversion element. Based on the frequency characteristics provided in the specifications for AD623, when the GB product is $800 \mathrm{kHz}$ and gain is 134, the upper limit of the band frequency will be $70 \mathrm{kHz}$. Since $70 \mathrm{kHz}$ is far higher than the sampling rate of $20 \mathrm{kHz}$ of the A/D converter, using the AD623 should pose no issues for this amplification circuit with respect to circuit design.

An angular velocity sensor, which relies on the principle that a vibrating body with angular velocity will exhibit the Coriolis force, was used to measure pitch angle. This compact piezoelectric vibrating gyro sensor features a simple cap-base structure measuring approximately $0.1 \mathrm{cc}$ in size and contains a ceramic bimorph vibrator. This gyro sensor was welded perpendicularly on the pin fixed to the moth. The pin doubled as a neutral point for the EMG amplification circuit. The LF411 operational amplifier was used with an amplification degree of 13 and zero bias being controlled using a variable resistor (Figure 5).

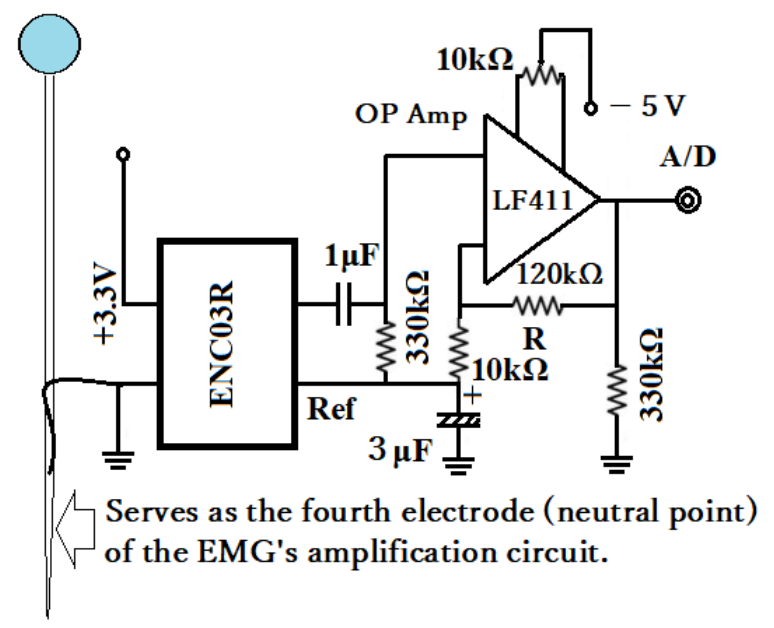

Fig. 5. Circuit diagram for angular velocity measurement using the Murata ENC03R [17].

In Figure 5, the pin inserted into the dorsal thorax is welded to the enameled wire and grounded and also serves as the fourth electrode of the EMG amplification circuit.

\subsection{Analog-to-digital conversion}

We recorded data to a PC using a 3-channel analog-todigital converter with a USB connection at a sampling rate of 20,000/sec and 16-bit resolution potential variation (y axis). We used Microsoft Excel to analyze the linear data, using programs for calculating EMG amplitude and PP intervals created with VBA.

\subsection{Results of experiment}

The data files for wing flapping for 100 moths consisted of recordings lasting 49 seconds at a sampling rate of $20 \mathrm{kHz}$ and 24 seconds at a sampling rate of $40 \mathrm{kHz}$. Since we processed the data in Excel, recording duration was restricted by the maximum number of rows permitted in an Excel sheet. The data obtained is explained below.

Figure 6 shows a characteristic 1-second section taken from 3 -channel data recorded for 49 seconds.

The data presented in Figure 6 corresponds to a 1second section illustrating the transition from a phase in $\mathrm{w}$ hich the moth slowly begins to flap its wings to the active flapping phase. As the amplitude of the pitch angle increas es, a transition from a double-peaked waveform to a singlepeak waveform occurs. The baselines (DC components) fo $r$ both the EMG1 (for the dorsal longitudinal muscle) and $t$ he EMG2 (for the dorsoventral muscle) switches to a decreasing trend at the transi tion. We believe this mode change in the DC component will be useful in assessing the relationship between the mo vement of the muscle as a whole and the depolarization of the inserted electrodes. The DC component cannot be obse 
rved with the AC amplification circuit due to capacitive co upling.

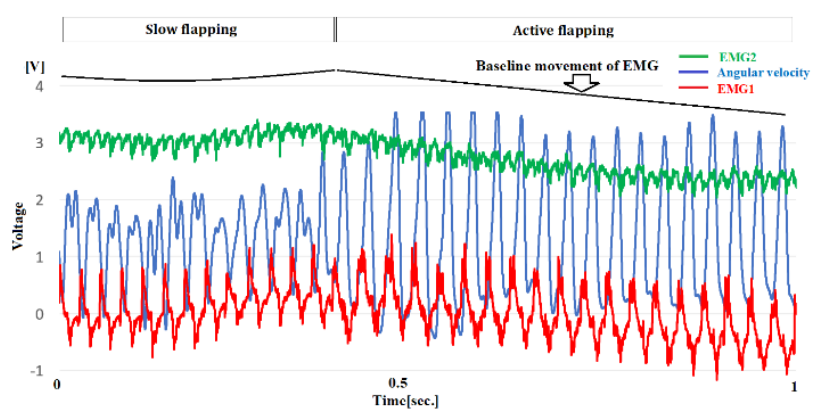

Fig. 6. Digital conversion performed at sampling rate of $20,000 / \mathrm{sec}$ and 16-bit potential variation (y axis) for 1 second.

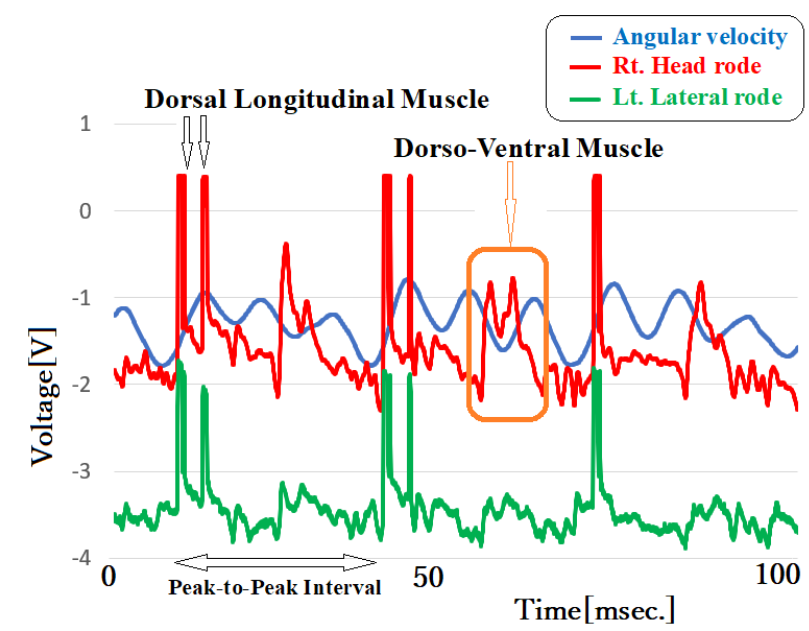

Fig. 7. Signals associated with DLM and DVM and PP intervals of EMG.

Figure 7 represents an enlarged 100-msec section of the plot above. We confirmed two peaks in the downstroke muscles, attributed to the difference in timing of the wingbeats for the left and right wings. The potential variation is most likely generated by the dorsal longitudinal muscle.

Between these peaks, there is a signal for the upstroke, which we believe is associated with the dorso-ventral muscle.

Figure 8 shows the data for 100 EMG amplitudes and the corresponding amplitudes of angular velocity. The correlation coefficient of the regression curve is $\mathrm{R}=0.792$, indicating generally high correlation (does not intentionally exclude outliers). This demonstrates that greater muscular strength correlates with greater angular velocity, suggesting that continuous EMG measurements can be used for physiological functional evaluations of moth flight.

Next, we analyzed frequency fluctuations. Figure 9 plots the data for 100 PP intervals and the amplitude of angular velocity for the corresponding intervals. The PP interval here is similar to the RR interval in ECG and represents the correlation between angular velocity and the interval between wingbeats.

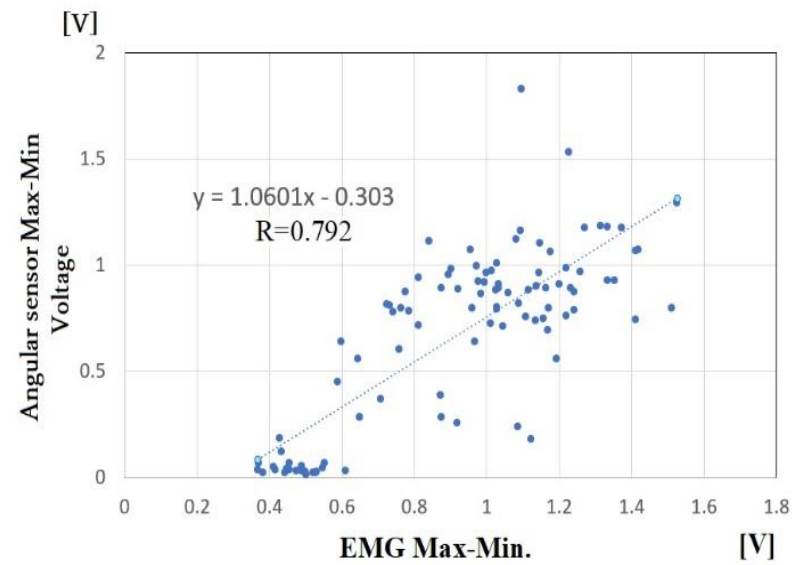

Fig. 8. Correlation between EMG and angular velocity.

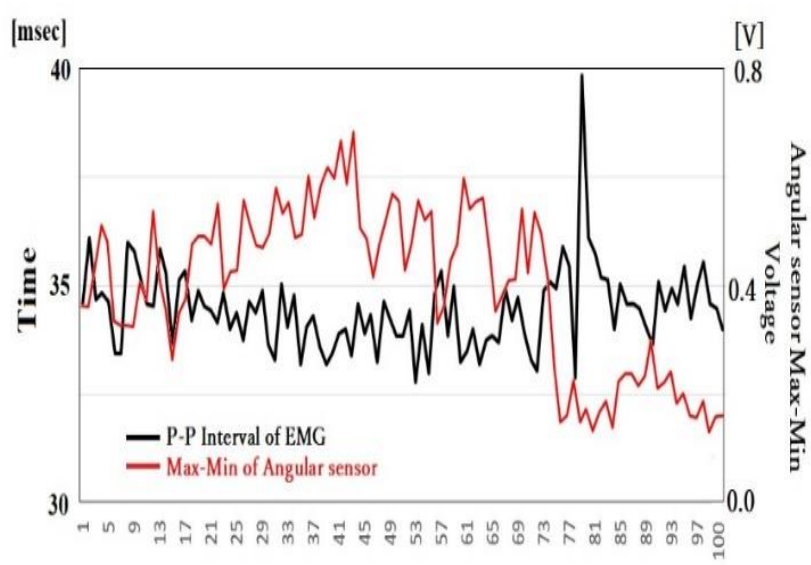

Fig. 9. 100 EMG amplitudes and corresponding angular velocity amplitudes.

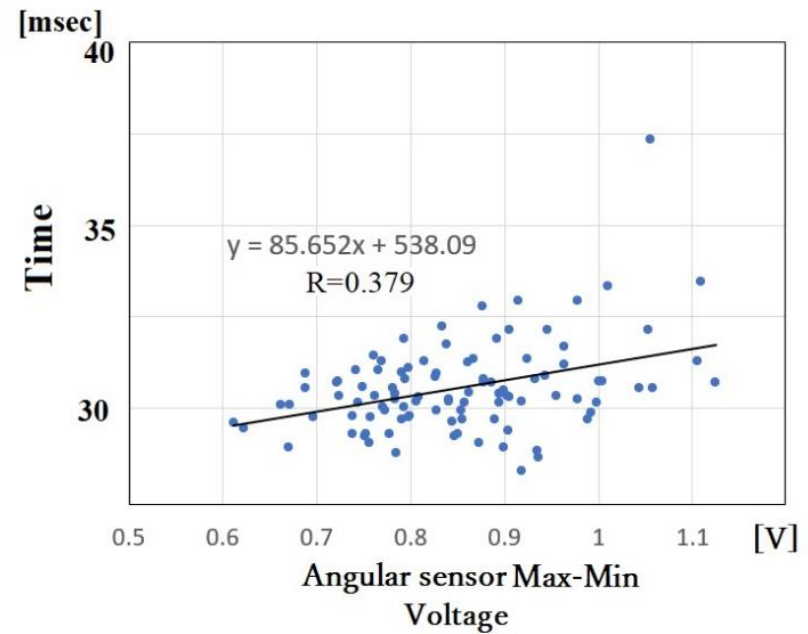

Fig. 10. Correlation between $100 \mathrm{PP}$ intervals and angular velocity amplitudes.

The correlation coefficient for the correlation between the fluctuation for the $100 \mathrm{PP}$ intervals and the amplitude of angular velocity is $\mathrm{R}=0.379$ shown in the Figure 10 . The 
accuracy of the regression curve is relatively poor (does not intentionally exclude outliers).

Although angular velocity is a factor that contributes to the fluctuation of the lift provided by wing flapping, our investigation showed a poor correlation between PP interval fluctuations of flapping motion and the fluctuation of the lift, suggesting that the former does not directly contribute to lift. This experiment failed to identify the physiological mechanism responsible for the PP interval fluctuations of flapping motion.

\section{DISCUSSIONS}

\subsection{Physiological functional evaluations of insects}

The experiment proved a correlation between angular velocity in the pitch direction of insects and EMG signals. Previous studies have analyzed insect flight using cameras and wind tunnels or EMG itself. However, these studies have been unable to establish a correlation between pitch angle and motor physiological data (corresponding to the EMG signal amplitude in this study). Our experiment corroborates this correlation and demonstrates the potential usefulness of EMG in functional evaluations of moths. Needless to say, physics supports the existence of a linear correlation between pitch angle and lift provided by wings. For agrochemicals, the exposure limits for chronic lowlevel exposure have been defined as 1/100 the LD50 value, the dose at which half of the exposed population dies in the case of acute exposure. No current standard for insects is based on physiological functional evaluations.

Even at doses far below 1/100 of the LD50 value, a decrease observed in EMG potential may indicate an onset of functional disorder. We believe this points to the potential for novel screening methods capable of evaluating the effects of repeated exposure to multiple agrochemicals. The application of such a method would not be limited to agrochemicals. It could also be used to assess exposure dose in radiation exposure incidents, such as the Fukushima-Daiichi nuclear power plant accident, based on functional evaluations of insects [18]-[24]. After the Fukushima-Daiichi nuclear power plant accident, professional journals reported numerous observations of anomalous white spots (depigmentation) on the wings of butterflies of the Lycaenidae family.

Government-specified exposure dose limits are calculated from the estimated total lifetime dose over 70 years and do not take into account functional evaluations of insects. As with LD50 values, calculations of these dose limits are not based on physiological evidence.

\section{2. $\Delta$ f fluctuations}

We confirmed a correlation between EMG amplitude and amplitude of angular velocity. What factor accounts for the fluctuations observed in PP intervals, a frequency component? We believe the answer will prove crucial in producing a parameter for screening functional disorders caused by chronic low-level exposure.

In human ECGs, the RR interval reflects the status of autonomic nervous system regulation. Similarly, we believe that fluctuations in the PP interval for wing flapping of the moth may reflect the state of regulation. We believe chronic low-level exposure to agrochemicals may manifest as anomalies in $\Delta \mathrm{f}$ fluctuations.

In the present experiment, we constrained the moths to keep them from ascending or flying forward, a factor that may have inhibited their free flapping motions. Additionally, our focus was on the two large muscles (DLM and DVM). We may have failed to make proper assessments of the movement of the two other relatively minor muscles, which are known to be associated with hovering and rotation in flight. Thus, the cause of $\Delta f$ fluctuations for these muscles may only be identified through monitoring of EMG during free flight.

In the next study, we expect to investigate the fluctuations of the two steering muscles (the 3rd axillary muscle and the subtrochanteric muscle) and fluctuations of PP-Interval, which are directly linked to the wings.

For subsequent experiments, we devised a transmitter using microchips and batteries for wrist watches $(1.5 \mathrm{~V})$ and carried out free-flight experiments after obtaining appropriate licenses for radio transmission from the Ministry of Internal Affairs and Communications. The experiment carried out by Prof. Ando's team, which preceded ours, achieved 2-channel transmission using an 80 $\mathrm{MHz}$ band FM radio transmitter [13]. We plan to pursue research on $\Delta f$ fluctuations to analyze the role of the remaining two muscles as part of efforts to establish a screening method for chronic low-level exposure.

\section{CONCLUSIONS}

Based on this study, we reached two conclusions (1 and 2 below). We also present two themes for future study, 3 and 4.

1. We observed a correlation coefficient $(\mathrm{R}=0.792)$ between the lift provided by wing flapping (estimated from angular velocity) and EMG signal amplitude.

2. The correlation coefficient for the correlation between PP intervals and lift (estimated from angular velocity) provided by wing flapping was $R=0.379$. Thus, based on 
the results of the present processing method, we conclude no correlation between the two can be observed.

3. The flapping mechanism appears to incorporate a dynamic gear change between different modes of muscle employment. This suggests we should consider nonlinear analytical techniques, such as the analysis of whip motion using the Lagrangian equation of motion.

4. If the $\Delta \mathrm{f}$ fluctuation can be measured in addition to EMG amplitude, it should be possible to evaluate the status of moth flight using the two parameters of potential and frequency axes. Physiological evaluations based on amplitude and frequency fluctuations may lead to an indicator that potentially replaces the LD50 value used to assess agrochemical toxicity.

\section{Acknowledgements}

We wish to thank Professor Noriyasu Ando of the Maebashi Institute of Technology, who provided the moths used in the present study. We also wish to thank the timely help given by Prof. Kiyoshi Kurokawa, the National Graduate Institute for Policy Studies, Professor Yoshiya Muraki of Seisa University for offering his guidance with our electronic circuits. Dr. Hiroshi Juzoji at EFL Inc. created a main portion of the analytical software.

\section{REFERENCES}

[1] R. Isenring, "Pesticides and the loss of biodiversity," Pesticide Action Network Europe, March 2010. https://www.paneurope.info/old/Resources/Briefings/Pesticides_and_t he_loss_of_biodiversity.pdf.

[2] Beyond pesticides, "Impacts of Pesticides on Wildlife," May 2020 , https://www.beyondpesticides.org/programs/wildlife.

[3] M. DiBartolomeis, S. Kegley, P. Mineau, R. Radford, K. Klein, "An assessment of acute insecticide toxicity loading (AITL) of chemical pesticides used on agricultural land in the United States," PLOS-ONE, August

2019. https://journals.plos.org/plosone/article?id=10.1371/j ournal.pone.0220029.

[4] J. Lundgren, S. Fausti, "Trading biodiversity for pest problems," Science Advances, Vol. 1, No. 6, e1500558. DOI: 10.1126/sciadv.1500558, 2015.

[5] K. Suzuki, T. Inamuro, "An improved lattice kinetic scheme for incompressible viscous fluid flows," Int. J. Mod. Phys. C 25. 1340017, 2014.

[6] M. Shindo, T. Fujikawa, K. Kikuchi, “Analysis of Roll Rotation Mechanism of the Butterfly for Development of a Small Flapping Robot," in Proceeding of the 3rd International Conference on Design Engineering and Science, pp. 90-96, 2014.

[7] F. Lehmann, S. Pick. "The aerodynamic benefit of wing- wing interaction depends on stroke trajectory in flapping insect wings," Journal of Experimental Biology, vol. 210, pp. 1362-1377, 2007.

[8] S. Hassler, "Winged Victory: Fly-Size Wing Flapper Lifts Off," IEEE Spectrum, https://spectrum.ieee.org/aerospace/aviation/wingedvictory-flysize-wing-flapper-lifts-off, 2008.

[9] T. Deora, N. Gundiah, S. Sane, "Mechanics of the thorax in flies," Journal of Experimental Biology, vol. 220, pp. 1382-1395, 2017.

[10] K. Nakada, J. Hata, "Development and physiological assessments of multimedia avian esophageal catheter system," Journal of Multimedia Information System, vol. 5, no. 2, pp. 121-130, 2018.

[11] I. Nakajima, H. Juzoji, K. Ozaki, N. Nakamura, "Communications Protocol Used in the Wireless Token Rings for Bird-to-Bird," Journal of Multimedia Information System, vol. 5, no. 3, pp. 163-170, 2018.

[12] K. Nakada, I. Nakajima, J. Hata, M. Ta, "Study on Vibration Energy Harvesting with Small Coil for Embedded Avian Multimedia Application," Journal of Multimedia and Information System, vol. 5, no. 1, pp. 47-52, 2018.

[13] N. Ando, I. Shimoyama, R. Kanzaki, “A dual-channel FM transmitter for acquisition of floght muscle activities from the freely flying hawkmoth, Agrius convolvuli," Journal of Neuroscience Methods, vol. 115, pp. 181-187, 2002

[14] M. Shimoda, M. Kiuchi, "Oviposition behavior of the sweet potato hornworm, Agrius convolvuli (Lepidoptera; Sphingidae), as analysed using an artificial leaf," Apply Entomol Zool, vol. 33, no. 4. pp. $525-534,1998$.

[15] A. Zagorinskii, O. Gorbunov, A. Sidorov, "An Experience of Rearing Some Hawk Moths (Lepidooptera, Sphingidae) on Artificial Diets," Entomological Review, vol. 93, no. 9, pp.1107-1115, 2013.

[16] OP AMP AD 623 Datasheet. https://www.analog.com/media/en/technicaldocumentation/data-sheets/AD623.pdf, May 2020.

[17] OP AMP LF411 Datasheet. https://newton.ex.ac.uk/teaching/CDHW/Electronics2 /DataSheets/LF411.pdf, May 2020.

[18] Agrius convolvuli, Wikipedia: https://en.wikipedia.org/wiki/Agrius_convolvuli. May 2020.

[19] K. Sakauchia, W. Tairaab, A. Hiyam, Tetsuji Imanaka, Joji M. Otaki, "The pale grass blue butterfly in exevacuation zones 5.5 years after the Fukushima nuclear accident: Contributions of initial high-dose exposure to transgenerational effects," J. of AsiaPacific Entomology, vol. 23, pp. 242-253, 2020.

[20] J. Otaki, "Fukushima's lessons from the blue butterfly: a risk assessment of the human living environment in the post-Fukushima era," Integr. Environ. Assess. Manag., vol. $\quad 12, \quad$ pp.667-672. https://doi.org/10.1002/ieam.1828, 2016.

[21] J. Otaki, W. Taira, "Current status of the blue butterfly in Fukushima research," Journal of Heredity, vol. 109, 
pp. 178-187. https://doi.org/10.1093/jhered/esx037, 2018.

[22] J. Otaki, A. Hiyama, A., M. Iwata, T. Kudo, "Phenotypic plasticity in the rangemargin population of the lycaenid butterfly Zizeeria maha," BMC Evol. Biol., vol. 10, pp. 252. https://doi.org/10.1186/14712148-10-252, 2018.

[23] J. Otaki, Understanding low-dose exposure and field effects to resolve the field laboratory paradox: multifaceted biological effects from the Fukushima nuclear accident, Awwad, N.S., AlFaify, S.A., (Eds.). New Trends in Nuclear Science, Intech Open, London. pp. 49-71. ISBN 978-1-78984-656-0. doi: 10.5772/intech Open. 79870, 2018.

[24] J. Otaki, W. Taira, "Current Status of the Blue Butterfly in Fukushima Research," Journal of Heredity, vol. 109, no. 2, pp.178-187, 2018.

\section{Authors}

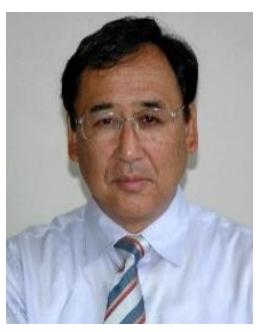

Isao Nakajima is a specially appointed professor of Seisa University and a visiting professor of Nakajima Labo. at the Dept. of Emergency Medicine and Critical Care, Tokai University School of Medicine. He got the Doctor of Applied Informatics (Ph.D.), Graduate School of Applied Informatics University of Hyogo 2009, and the Doctor of Medicine (Ph.D.), Post Graduate School of Medical Science Tokai University 1988, and the Medical Doctor (M.D.) from Tokai University School of Medicine 1980. He has been aiming to send huge multimedia data from moving ambulance via communications satellite to assist patient's critical condition. A board member of the Pacific Science Congress, a Rapporteur for eHealth of ITU-D SG2.

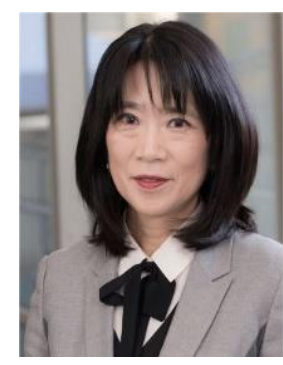

Yukako Yagi is at the Digital Pathology Laboratory of the Josie Robertson Surgical Center serves as an incubator to explore, evaluate and develop new technology to advance digital pathology in a clinical setting and actively engage vendors to help improve the technology and develop clinical applicability. Collaborations with clinical departments (e.g., Surgery), Radiology, Medical Physics, and Informatics groups, enhance these assessments and creates opportunities for multidisciplinary applications. She completed her Doctorate in Medical Science at Tokyo Medical University in Japan. She has a broad interest in various aspects of medical science, which include the development and validation of technologies in digital imaging, such as color and image quality calibration, evaluation and optimization, digital staining, 3D imaging, and decision support systems for pathology diagnosis, research and education.

Since joining MSK, she has led pioneering work using MicroCT, Whole Slide Imaging (WSI) and Confocal imaging to connect multi-dimensional and multi-modality images (e.g., single-cell to whole-body analysis). She participated in creating image viewers for several imaging modalities and established new imaging data formats. Her team has established the technology to streamline colorization within Pathology 3D, i.e., H\&E, immunoflorescent, immuno-histochemistry, and florescence in situ hybridization imaging. Once the colorization is mapped, it can be correlated with another modality such as radiology, for a holistic analysis that will improve patient care and outcomes. The team also created 3D histology images of a single organ using thousands of slides.

Her works further enriches our knowledge of disease by integrating computational pathology data with other specimenrelated data (genomics, proteomics, radiographic imaging, etc.). This brings an unprecedented breadth and depth of information to each individual case and yields a comprehensive, multidimensional analysis that would otherwise be impossible. 